Full length article

\title{
Deciphering the combined effect of bone morphogenetic protein 6 and calcium phosphate on bone formation capacity of periosteum derived cells-based tissue engineering constructs
}

\author{
Wei Ji $^{\text {a,b,1 }}$, Greet Kerckhofs ${ }^{\text {a,b,c,1 }}$, Carla Geeroms ${ }^{\text {a,b }}$, Marina Marechal ${ }^{\text {a,b }}$, Liesbet Geris ${ }^{\text {a,d,2, }}$ \\ Frank P. Luyten ${ }^{\mathrm{a}, \mathrm{b}, 2, *}$ \\ a Prometheus, Division of Skeletal Tissue Engineering, KU Leuven, Leuven, Belgium \\ ${ }^{\mathrm{b}}$ Skeletal Biology and Engineering Research Center, Department of Development and Regeneration, KU Leuven, Leuven, Belgium \\ ${ }^{\mathrm{c}}$ Biomechanics Lab, Institute of Mechanics, Materials, and Civil Engineering, UCLouvain, Belgium \\ ${ }^{\mathrm{d}}$ Biomechanics Research Unit, GIGA In silico Medicine, University of Liege, Liege, Belgium
}

\section{A R T I C L E I N F O}

\section{Article history:}

Received 25 July 2018

Received in revised form 18 September 2018

Accepted 25 September 2018

Available online $\mathrm{xxxx}$

\section{Keywords:}

Bone tissue engineering

Periosteal cells

Calcium phosphate

Cell signaling

BMP

Bone forming capacity

\begin{abstract}
A B S T R A C T
Cell based combination products with growth factors on optimal carriers represent a promising tissue engineering strategy to treat large bone defects. In this concept, bone morphogenetic protein (BMP) and calcium phosphate ( $\mathrm{CaP}$ )-based scaffolds can act as potent components of the constructs to steer stem cell specification, differentiation and initiate subsequent in vivo bone formation. However, limited insight into BMP dosage and the cross-talk between BMP and CaP materials, hampers the optimization of in vivo bone formation and subsequent clinical translation. Herein, we combined human periosteum derived progenitor cells with different doses of BMP6 and with three types of clinical grade CaP-scaffolds (ChronOs ${ }^{\circledR}$, ReproBone ${ }^{\mathrm{TM}}$, \& CopiOs $^{\circledR}$ ). Comprehensive cellular and molecular analysis was performed based on in vitro cell metabolic activity and signaling pathway activation, as well as in vivo ectopic bone forming capacity after 2 weeks and 5 weeks in nude mice. Our data showed that cells seeded on CaP scaffolds with an intermediate $\mathrm{Ca}^{2+}$ release rate combined with low or medium dosage of BMP6 demonstrated a robust new bone formation after 5 weeks, which was contributed by both donor and host cells. This phenomenon might be due to the delicate balance between $\mathrm{Ca}^{2+}$ and BMP pathways, allowing an appropriate activation of the canonical BMP signaling pathway that is required for in vivo bone formation. For high BMP6 dosage, we found that the BMP6 dosage overrides the effect of the $\mathrm{Ca}^{2+}$ release rate and this appeared to be a dominant factor for ectopic bone formation. Taken together, this study illustrates the importance of matching BMP dosage and CaP properties to allow an appropriate activation of canonical BMP signaling that is crucial for in vivo bone formation. It also provides insightful knowledge with regard to clinical translation of cell-based constructs for bone regeneration.
\end{abstract}

\section{Statement of Significance}

The combination of bone morphogenetic proteins (BMP) and calcium phosphate (CaP)-based biomaterials with mesenchymal stromal cells represents a promising therapeutic strategy to treat large bone defects, an unmet medical need. However, there is limited insight into the optimization of these combination products, which hampers subsequent successful clinical translation. Our data reveal a delicate balance between $\mathrm{Ca}^{2+}$ and BMP pathways, allowing an appropriate activation of canonical BMP signaling required for in vivo bone formation. Our findings illustrate the importance of matching BMP dosage and CaP properties in the development of cell-based constructs for bone regeneration.

(c) 2018 Acta Materialia Inc. Published by Elsevier Ltd. All rights reserved.

\footnotetext{
* Corresponding author at: Herestraat 49, box 7003, 3000 Leuven, Belgium. E-mail address: frank.luyten@uzleuven.be (F.P. Luyten).

1 These authors contributed equally to this work.

2 These authors share senior authorship.
}

\section{Introduction}

Long-bone fractures are common and are one of the most frequent non-fatal injuries sustained following trauma worldwide 
[1-3]. In the United States, almost 8 million fractures are reported each year, and $5-10 \%$ of fractures fail to heal properly, resulting in delayed or non-unions $[2,4]$. As populations age, the incidence of non-unions also increase, owing to comorbidities such as diabetes [5]. The current state of the art to treat non-union fractures involves rigid fixation of the fractures and transplantation of autologous or allogenic bone grafts to stimulate the bone growth [6]. However, the inherent drawbacks of this approach, including insufficient autologous resources, pain and donor site morbidity, strongly urge clinicians and researchers to explore alternative therapeutic strategies.

With the advances of cell biology and material sciences, several alternative strategies are emerging to treat non-union fractures. One of these promising strategies is to develop advanced therapeutic medicinal product (ATMP) with stem cells laden in optimal carrier materials $[3,7,8]$. By using stem cells to initiate fracture healing and carrier materials (i.e., scaffolds) to guide and stimulate bone tissue formation [3,9], such cell-based ATMPs are envisioned to overcome the limited endogenous regenerative capacity of the patients $[3,8]$.

Human periosteum derived cells (hPDCs) are of interest for bone regeneration owing to their key roles in fracture healing [10-13]. Moreover, recent studies demonstrate that postnatal hPDCs exhibit greater clonogenicity, differentiation capacity, and bone regenerative potential after transplantation compared to bone marrow stromal cells, although these two types of cells are derived from a common embryonic mesenchymal lineage [14]. On the other hand, calcium phosphate $(\mathrm{CaP})$ materials have been widely used as synthetic bone substitutes in view of their excellent osteoconductivity as well as biodegradability $[8,15,16]$. Currently, the most frequently used clinically available CaP materials are hydroxyapatite (HA), $\beta$-tri-calcium phosphate ( $\beta$-TCP) and combinations of HA and $\beta$-TCP, which display distinctive $\mathrm{Ca}^{2+}$ release rates due to their different $\mathrm{Ca} / \mathrm{P}$ atomic ratio [17]. Our previous data has shown that the sufficient release of $\mathrm{Ca}^{2+}$ was an essential determinant for inducing osteogenic differentiation of hPDCs [18-20]. Furthermore, the chemical and structural properties of $\mathrm{CaP}$ also played an important role in determining the in vivo bone formation capacity of hPDCs/CaP constructs [21]. It is also important to note that bone formation in the aforementioned cell-based CaP constructs is a slow process ( 8 weeks) and until today, only limited amounts of bone and marrow formation have been obtained. Hence, the addition of osteoinductive factors such as bone morphogenetic proteins (BMPs) appears to be a straightforward approach to improve the bone forming capacity of hPDC/CaP constructs.

BMP2 (Infuse ${ }^{\circledR}$ or InductOs ${ }^{\mathrm{TM}}$ ) and BMP7 (Osigraft ${ }^{\circledR}$ ) have been widely introduced to the clinic to treat non-healing bone defects or complicated bone fractures [22,23]. However, both BMP2 and BMP7 have been confronted with major issues such as early osteolysis, significant inflammation, heterotopic ossification, leading to less robust outcomes in clinics and limited use in the postmarketing period [24]. Recently, BMP6 has emerged as a unique and important BMP family member during skeletal development. Previous studies showed that BMP6 was more efficient in driving osteoblast differentiation of mesenchymal stem cells than BMP2 and BMP7 [25]. Vukicevic et al [24] reported that at 3 months of age, BMP6-/- mice showed 32\% decrease of trabecular bone volume, and such effects could not be overcome by increased expression of other BMPs. Our previous results have also shown that exogenous BMP6 coated calcium phosphate scaffolds induced subcutaneous bone formation after 5 weeks, and there was a dominant role of BMP6 over the material in such ectopic bone formation process [26]. Although numerous studies have been performed to investigate biological functions of BMP6, the selection of BMP6 dosage is mainly experienced-based. Furthermore, the combined effects of BMP6 (at different dosages) and CaP properties (at different $\mathrm{Ca}^{2+}$ release rates) on cell behavior, as well as on in vivo ectopic bone forming capacity are still largely unknown.

This study is aimed to decipher the importance and influence of BMP6 dosage and CaP properties, as well as their combination, on the biological performance of cell-based tissue engineering (TE) constructs. For this purpose, hPDCs were seeded into three different clinical-grade CaP-based scaffolds (with distinctly different $\mathrm{Ca}^{2+}$ release rates) combined with three doses of BMP6. The obtained constructs were evaluated for in vitro cell metabolic activity and signal activation after $16 \mathrm{~h}$ prior to implantation, as well as in vivo ectopic bone forming capacity after 2 weeks and 5 weeks after implantation. Finally, a multivariate statistical analysis was used to deduce key factors in the empirical data set. Our hypothesis was that the interactions between $\mathrm{Ca}^{2+}$ release rate and BMP6 dosage affect the in vitro cell behavior, hence influencing the in vivo skeletal tissue formation of hPDCs seeded TE constructs.

\section{Materials and methods}

\subsection{Scaffolds preparation}

Three different commercially available and clinically approved CaP-based materials were used, namely ReproBone ${ }^{\mathrm{TM}}$ (Ceramysis, Sheffield, England), ChronOS ${ }^{\circledR}$ (Depuy Synthes, Oberdorf, Switzerland), and CopiOs ${ }^{\circledR}$ (Zimmer Inc, Warsaw Indiana, USA). The characteristics and the composition of these materials, as provided by the supplier, can be found in Table 1 . For each type of material, scaffolds ( $3 \mathrm{~mm} \times 3 \mathrm{~mm} \times 3 \mathrm{~mm}$ ) were cut using a scalpel and a ruler. All preparations and handling of the scaffolds were accomplished in a sterile environment.

\subsection{Expansion of human periosteum-derived cells (hPDCs)}

Isolation and in vitro expansion of hPDCs were carried out as previously described $[12,26]$. The ethical committee for human medical research (KU Leuven) approved all procedures, and the patient informed consents were obtained. In brief, expanded cells from 4 different donors ( $29 \pm 12$ years old, detailed information in Supplementary Table S1) were pooled as a representative sample and expanded in vitro in culture media [DMEM-c; consisted of Dulbecco's Modified Eagle Medium (DMEM GlutaMax ${ }^{\mathrm{TM}}$, Invitrogen, Merelbeke, BE) supplemented with $10 \%$ Fetal Bovine Serum (Gibco, Merelbeke, BE), 1\% antibiotics/antimycotics, and 1\% sodium pyruvate (Invitrogen)].

\subsection{Cell seeding and BMP coating of scaffolds}

Recombinant human BMP6 was a generous gift provided by Prof. Slobodan Vukicevic (Centre for Translational and Clinical Research, University of Zagreb, Croatia). Upon confluence, hPDCs (Passage 7) were trypsinized, counted and re-suspended in phosphate buffered saline (PBS, pH 7.4, Gibco). Afterwards, $20 \mu \mathrm{L}$ of cell suspension containing different dosage of BMP6 (ranging from $0 \mathrm{ng} / \mathrm{mm}^{3}$ to $210 \mathrm{ng} / \mathrm{mm}^{3}$ ) were drop seeded onto the aforementioned scaffolds that had been pre-wet with $1 \mathrm{~mL}$ of PBS. The loading dosage of cells and BMP6 were normalized to the open volume and the total volume space of the scaffold types, respectively, which were determined from the microcT-based image analysis in our previous work [21]. Table 2 listed the precise information of cell seeding density and BMP6 dosage for all the conditions. For samples loaded only with BMP6, $20 \mu \mathrm{L}$ of PBS containing the equivalent amount of BMP6 was drop seeded into the scaffolds. After $1 \mathrm{~h}$ of incubation at $37{ }^{\circ} \mathrm{C}, 5 \% \mathrm{CO}_{2}$ and $95 \%$ relative humidity, $4.5 \mathrm{~mL}$ of DMEM-c was added. The seeded scaffolds were 
Table 1

Composition and characteristics of different materials as provided by suppliers and our previous scanning [21].

\begin{tabular}{|c|c|c|c|c|c|}
\hline Scaffold & Supplier & CaP chemistry & Matrix & Total volume $\left(\mathrm{mm}^{3}\right)$ & Open volume $\left(\mathrm{mm}^{3}\right)$ \\
\hline ChronOs $^{\circledR}$ & Depuy Synthes & $100 \% \beta-\mathrm{Ca}_{3}\left(\mathrm{PO}_{4}\right)_{2}$ & N.A & 27 & 17.55 \\
\hline ReproBone $^{\mathrm{TM}}$ & Ceramysis & $\mathrm{Ca}_{5}\left(\mathrm{PO}_{4}\right)_{3}(\mathrm{OH}) / \beta-\mathrm{Ca}_{3}\left(\mathrm{PO}_{4}\right)_{2}(60 \% / 40 \%)$ & N.A & 27 & 20.08 \\
\hline CopiOs $^{\circledR}$ & Zimmer & $\mathrm{CaHPO} 4$ & Lyophilized bovine collagen I & 27 & 21.78 \\
\hline
\end{tabular}

Table 2

Cell seeding density and BMP6 loading amount for different scaffolds.

\begin{tabular}{|c|c|c|c|c|}
\hline & & ChronOs $^{\circledR}$ & ReproBone $^{\mathrm{TM}}$ & CopiOs $^{\circledR}$ \\
\hline \multirow{2}{*}{\multicolumn{2}{|c|}{$\begin{array}{l}\text { Cell seeding density (normalized to the open volume per scaffold) } \\
\text { Theoretical cell amount per scaffold }\end{array}$}} & $50,000 / \mathrm{mm}^{3}$ & $50,000 / \mathrm{mm}^{3}$ & $50,000 / \mathrm{mm}^{3}$ \\
\hline & & $0.9 \times 10^{6}$ & $1.0 \times 10^{6}$ & $1.1 \times 10^{6}$ \\
\hline \multirow{4}{*}{ BMP6 loading amount (normalized to the total volume per scaffold) } & None & $0 \mathrm{ng} / \mathrm{mm}^{3}$ & $0 \mathrm{ng} / \mathrm{mm}^{3}$ & $0 \mathrm{ng} / \mathrm{mm}^{3}$ \\
\hline & Low & $30 \mathrm{ng} / \mathrm{mm}^{3}$ & $30 \mathrm{ng} / \mathrm{mm}^{3}$ & $30 \mathrm{ng} / \mathrm{mm}^{3}$ \\
\hline & Medium & $120 \mathrm{ng} / \mathrm{mm}^{3}$ & $120 \mathrm{ng} / \mathrm{mm}^{3}$ & $120 \mathrm{ng} / \mathrm{mm}^{3}$ \\
\hline & High & $210 \mathrm{ng} / \mathrm{mm}^{3}$ & $210 \mathrm{ng} / \mathrm{mm}^{3}$ & $210 \mathrm{ng} / \mathrm{mm}^{3}$ \\
\hline \multirow{4}{*}{ Theoretical BMP6 loading amount per scaffold } & None & 0 ng & $0 \mathrm{ng}$ & $0 \mathrm{ng}$ \\
\hline & Low & $810 \mathrm{ng}$ & $810 \mathrm{ng}$ & $810 \mathrm{ng}$ \\
\hline & Medium & 3240 ng & 3240 ng & 3240 ng \\
\hline & High & $5670 \mathrm{ng}$ & $5670 \mathrm{ng}$ & $5670 \mathrm{ng}$ \\
\hline
\end{tabular}

incubated overnight (approximal $16 \mathrm{~h}$ ) to allow cell attachment before further in vitro and in vivo evaluation. The detailed information for all the experimental groups and sample preparation are listed in Table 3.

\subsection{Lactate and glucose measurements}

Part of the media from the cell-laden samples was collected to assess the lactate and glucose concentrations using two clinical diagnosis test strips; lactate Pro ${ }^{\mathrm{TM}}$ test strip (ARKRAY, Europe B. $\mathrm{V}, \mathrm{NL}$ ) and Contour ${ }^{\circledR}$ blood glucose test strips (Bayer Consumer Care AG, $\mathrm{CH}$ ), respectively, as described in our previous study [26]. Each sample was measured twice to minimize technical error. Blank medium was measured as control to calculate lactate production and glucose consumption. Thereafter, the ratio of lactate production to glucose consumption per cell was calculated to compare the metabolic activity of the cells upon seeding in different conditions.

\subsection{Western blotting}

Western blotting of the cell-laden samples was performed to assess the downstream pathway activation similar as our previous report [26]. Protein was extracted from cell-seeded scaffolds using Cell Extraction Buffer (Thermo Scientific) containing 0.3 M Phenylmethanesulphonyl fluoride and Protease Inhibition Cocktail (Sigma-Aldrich, Bornem, BE). Protein concentration was determined using the Pierce BCA Protein Assay Kit (Thermo Scientific) according to manufacturer's instructions. $15 \mu \mathrm{g}$ of cell protein was loaded into and separated using a NuPAGE 4-12\% Bis-Tris gel (Invitrogen). These proteins were subsequently electrotransferred to a Polyvinylidene fluoride membrane by semi-dry transfer (Invitrogen). The membranes were blocked with 5\% BSA solution for $30 \mathrm{~min}$ followed by an overnight incubation with primary antibodies at $4{ }^{\circ} \mathrm{C}$. The following primary antibodies were used: rabbit monoclonal phospho-SMAD1/5 (1:1000, Cell Signaling \#9516), rabbit monoclonal SMAD1 (1:1000, Cell Signaling\#6944), rabbit monoclonal phospho-Erk1/2 (1:1000, Cell Signaling \#4377), rabbit monoclonal Erk1/2 (1:1000, Cell Signaling \#4695) rabbit monoclonal phospho-p38 MAPK (1:1000, Cell Signaling \#4511), rabbit monoclonal p38 MAPK (1:1000, Cell Signaling \#8690), mouse monoclonal to GAPDH (1:1000, Cell Signaling \#97166). The corresponding HRP-conjugated secondary antibodies were used at a dilution of 1:2000 (Jackson, Pennsylvania, US) and images were developed by a LAS3000 Imaging System (FUJI) following the application of SuperSignal ${ }^{\circledR}$ West Femto reagent (Thermo Scientific, Illnois, US). Densitometry analysis, standardized to GAPDH as a control for protein loading, was carried out with ImageJ software (National Institutes of Health, USA). Triplicate samples were assessed for each condition $(\mathrm{n}=3)$.

\subsection{Subcutaneous implantation}

In total, 24 NMRI-nu/nu mice (female, 8 weeks old) were used in the current study, and all procedures were performed according to the approved guidelines of the local ethical committee for Animal Research (KU Leuven). The obtained scaffolds as described in Section 2.3. were randomly implanted subcutaneously in the shoulder and the back area at the cervical region of animals. The remaining medium of the implanted scaffolds was collected and centrifuged. The pellet was then resuspended in RLT buffer (Qiagen, Venlo, The Netherlands) and stored at $-80^{\circ} \mathrm{C}$ for DNA quantification (Qubit system, Invitrogen, Belgium) to determine the cell seeding efficiency. After 2 weeks and 5 weeks of implantation, the mice were sacrificed and the implants were collected. Each explant was then fixed in $4 \%$ paraformaldehyde for $2 \mathrm{~h}$ and stored in PBS at $4{ }^{\circ} \mathrm{C}$ until further analyses.

\subsection{Microfocus computed tomography and 3D image analysis}

After 5 weeks of implantation, all explants were scanned using microCT. We applied a Phoenix NanoTom S (GE Measurement and Control Solutions, Germany) with a $180 \mathrm{kV} / 15 \mathrm{~W}$ highperformance microfocus X-ray tube. An operation voltage of $60 \mathrm{kV}$ was used, along with a current of $162 \mu \mathrm{A}$ and a $0.3 \mathrm{~mm}$ filter of aluminum. We used an exposure time of $500 \mathrm{~ms}$ with a frame averaging of 1 and an image skip of 0 (i.e. fast scanning mode), and we acquired 2400 radiographs per sample, resulting in a scanning time of $20 \mathrm{~min}$. The applied isotropic voxel size was $3 \mu \mathrm{m}$.

As described in detail in previous work [21], prior to the 3D morphometric analysis, the explant was outlined automatically by a region of interest (ROI) using an in-house developed MatlabTM tool (MorphingCiTy - ULg, Liege, Belgium). A three-level Otsu segmentation algorithm was used for segmenting bone from the CaP scaffold material (three classes of voxels i.e. background, bone and $\mathrm{CaP}$ ). The binarized images were then denoised (closing 
Table 3

Experiemtal design and group conditions.

\begin{tabular}{|c|c|c|c|c|}
\hline & Group $^{b}$ & Evaluation & Investigation period & Analysis methods \\
\hline Cell-laden samples ${ }^{a}$ & $\begin{array}{l}\text { ChronOs_c_none } \\
\text { ChronOs_c_low } \\
\text { ChronOs_c_medium } \\
\text { ChronOs_c_high } \\
\text { ReproBone_c_none } \\
\text { ReproBone_c_low } \\
\text { ReproBone_c_medium } \\
\text { ReproBone_c_high } \\
\text { CopiOs_c_none } \\
\text { CopiOs_c_low } \\
\text { CopiOs_c_medium } \\
\text { CopiOs_c_high }\end{array}$ & In vitro $\&$ in vivo & $\begin{array}{l}\text { In vitro: } 16 \mathrm{~h}(\mathrm{n}=3) \\
\text { In vivo: } 2 \text { weeks }(\mathrm{n}=3) \& 5 \text { weeks }(\mathrm{n}=3)\end{array}$ & $\begin{array}{l}\text { In vitro: } \\
\text { - lactate and glucose measurements } \\
\text { - western blotting } \\
\text { In vivo: } \\
\text { - } \mu \mathrm{CT} \\
\text { - (immuno)histology }\end{array}$ \\
\hline Cell-free samples & $\begin{array}{l}\text { ChronOs_low } \\
\text { ChronOs_medium } \\
\text { ChronOs_high } \\
\text { ReproBone_low } \\
\text { ReproBone_medium } \\
\text { ReproBone_high } \\
\text { CopiOs_low } \\
\text { CopiOs_medium } \\
\text { CopiOs_high }\end{array}$ & In vivo & In vivo: 2 weeks $(\mathrm{n}=3)$ \& 5 weeks $(\mathrm{n}=3)$ & $\begin{array}{l}\text { In vivo: } \\
\quad \bullet \mu \mathrm{CT} \\
\cdot(\text { immuno)histology }\end{array}$ \\
\hline
\end{tabular}

\footnotetext{
a For cell-laden samples, cell seeding density is $50,000 / \mathrm{mm}^{3}$, normalized to the open volume per scaffold as shown in Table 2 .

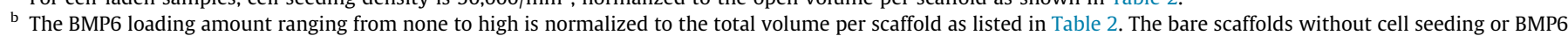
loading, without ectopic bone formation capacity as shown from our previous studies [21,26], were not included in this study.
}

operation of 1 , followed by despeckling of white and black speckles smaller than 200 voxels in 3D) and 3D morphometric analysis was done within the ROI using CTAn software (Bruker Micro-CT, Kontich, Belgium). We calculated the volume of bone in the total explant space as defined by the ROI as well as inside the scaffold only (defined by the borders of the scaffold material).

\subsection{General histology E immunohistochemical staining}

All explants were decalcified in an ethylene diamine tetraacetic acid (EDTA)/PBS solution ( $\mathrm{pH} \mathrm{7.5)} \mathrm{for} 14$ days and then embedded in paraffin. Sections ( $5 \mu \mathrm{m}$ thickness) were prepared using a microtome (Leica RM 2165, Leica Microsystems, Nussloch, Germany). The sections were stained with haematoxylin-eosin (HE) to assess the general histology. In addition, immunohistochemistry of human specific osteocalcin (hOCN) was performed to determine contribution of human donor cells to the new bone formation, as previously described [26]. In brief, the deparaffinized sections were quenched in $3 \% \mathrm{H}_{2} \mathrm{O}_{2}$ followed by antigen retrieval in sodium citrate, $10 \mathrm{mM}, \mathrm{pH} 6$, at $98^{\circ} \mathrm{C}$. Subsequently, sections were incubated with a primary anti-OCN guinea pig antibody at (1:1000, a generous gift from Dr. E. Van Herck, Legendo, KU Leuven) over night at $4{ }^{\circ} \mathrm{C}$. A HRP-conjugated anti guinea pig secondary antibody was used at a dilution of 1:500 (Jackson) and 3,3'diaminobenzidine (DAB, Sigma-Aldrich) were used as a chromogenic substrate. Sections were photographed with a digital camera equipped microscope (IX83-P22F, Olympus).

\subsection{Statistical analysis}

Data are expressed as individual data points with bars representing the average value. Statistical analysis was performed using Graphpad Prism v.6 software. Analysis of variance (ANOVA) with a Tukey post hoc test was used for all multiple group experiments. Statistical significance is indicated on all graphs as follows: ${ }^{*} \mathrm{p}<0.05,{ }^{* *} \mathrm{p}<0.01,{ }^{* * *} \mathrm{p}<0.001,{ }^{* * * *} \mathrm{p}<0.0001$ and $\mathrm{n}=3$ if nothing else is indicated. Hierarchical clustering using Ward's method and principle component analysis (PCA) were performed in JMP 14.0 (SAS Institute Inc., Cary, NC).

\section{Results}

\subsection{Cell metabolic activity}

Glucose metabolism was investigated by measuring the ratio of lactate production to glucose consumption in conditioned media after $16 \mathrm{~h}$ in vitro culture (Fig. 1). Regardless of BMP6 dosage, the conversion rate of glucose to lactate almost reached two when cells were seeded in ChronOs ${ }^{\infty}$, indicating the occurrence of anaerobic glycolysis. In contrast, the conversion of glucose to lactate significantly decreased when cells were seeded in ReproBone ${ }^{\mathrm{TM}}$ and CopiOs ${ }^{\infty}$. Particularly, the conversion ratio of glucose to lactate dropped to one when cells were seeded in CopiOs ${ }^{\infty}$, suggesting the occurrence of aerobic glycolysis (Fig. 1). The absolute concentrations of lactate production and glucose consumption per cell after $16 \mathrm{~h}$ in vitro culture are shown in Fig. S1.

\subsection{Signal activation by western blotting}

Next, protein lysates were prepared from cell-laden samples $16 \mathrm{~h}$ post seeding. Western blotting was performed to investigate canonical BMP-signaling (p-SMAD1/5) and non-canonical BMPsignaling (p-Erk \& p-p38) downstream pathways (Fig. 2). Phosphorylation of SMAD1/5 was observed from three types of scaffolds laden with BMP6, however, cells seeded in scaffolds with medium or slow $\mathrm{Ca}^{2+}$ release rate (ChronOs ${ }^{\circledR}$ and ReproBone ${ }^{\mathrm{TM}}$, respectively) showed higher phosphorylation of SMAD1/5 than the one with fast $\mathrm{Ca}^{2+}$ release rate $\left(\mathrm{CopiOs}^{\circledR}\right.$ ) when loaded with the same dosage of BMP6 (Fig. 2). Cells seeded in BMP6 loaded ChronOs ${ }^{\circledR}$ and ReproBo$\mathrm{ne}^{\mathrm{TM}}$ tend to reached their peak of phosphorylation of p-SMAD1/5 (approx. two-fold) when loaded with a medium BMP6 dosage (Fig. 2). In comparison, cells seeded in BMP6 loaded CopiOs ${ }^{\circledR}$ showed a significant increase of p-SMAD only when BMP6 dosage exceeded medium dosage, and the phosphorylation of SMAD1/5 exhibited a linear tendency with the increase of BMP6 loading dosage (Fig. 2).

All the conditions showed an evident phosphorylation of ErK1/2 MAPK (Fig. 2), and cells seeded in CopiOs ${ }^{\circledR}$, regardless of BMP6 loading dosage, showed a significant higher phosphorylation of Erk1/2 than the ones seeded in ChronOs $^{\circledR}$ and ReproBone ${ }^{\mathrm{TM}}$ 


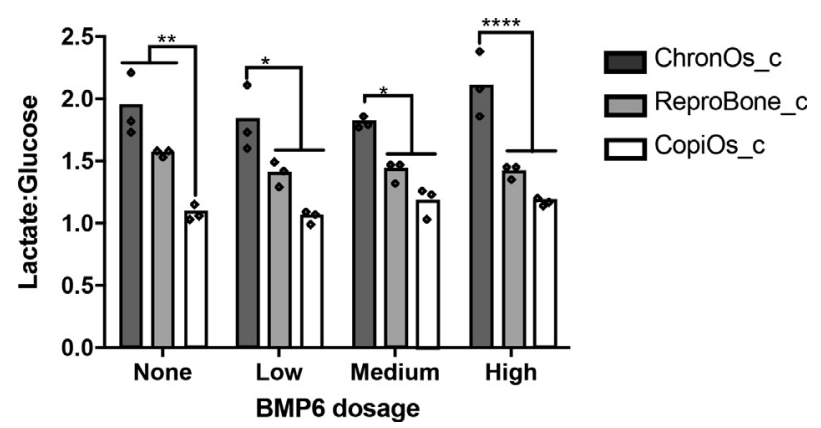

Fig. 1. Lactate/glucose ratio from cell-laden samples after $16 \mathrm{~h}$ in vitro culture.

(Fig. 2). On the other hand, cells seeded in CopiOs ${ }^{\circledR}$ showed a significantly higher phosphorylation of p38 MAPK compared to the ones seeded on ChronOs ${ }^{\circledR}$ and ReproBone ${ }^{\mathrm{TM}}$. It is also noted that the phosphorylation of $\mathrm{p} 38$ tends to decrease when a high dosage of BMP6 was applied in the three types of scaffolds (Fig. 2).

\subsection{Cell seeding efficiency for in vivo implants}

Cell-laden constructs loaded with different doses of BMP6 were subcutaneously implanted to evaluate their ectopic bone formation capacity. The average cell seeding efficiency for ChronOs ${ }^{\circledR}$ and CopiOs ${ }^{\circledR}$ was $75.5 \pm 10 \%$ and $82.1 \pm 4.95 \%$, respectively, regardless of BMP6 loading dosage. (Fig. S2). It was noted that the average cell seeding efficiency for ReproBone ${ }^{\mathrm{TM}}$ decreased to $64.17 \pm 13.09 \%$, although there was no significant statistical difference compared to the other two types of scaffolds (Fig. S2).

\subsection{Quantification of in vivo bone formation by microCT analysis}

After 5 weeks subcutaneous implantation, hPDCs seeded on scaffolds without loading BMP6 hardly induced bone formation (Fig. 3). Instead, newly formed bone both inside and outside the scaffolds was only visible when BMP6 was applied (Fig. 3). With a low dosage of BMP6, evident bone formation was observed with hPDCs seeded in ChronOs $^{\circledR}$ and ReproBone ${ }^{\mathrm{TM}}$ (Fig. 3). Yet, in CopiOs $^{\circledR}$ only one of the triplicates showed a small amount of newly formed bone (Fig. 3). There was a clear trend in all three types of scaffolds that both the total volume of new bone and bone volume outside the scaffolds increased along with the increasing BMP6 dosage (Fig. 3). When a high dosage of BMP6 was applied, there was no statistical difference between scaffolds in terms of total volume of new bone formation (Fig. 3). Furthermore, the new bone formed inside the scaffolds was independent of BMP6 dosage but strongly influenced by the different types of scaffolds. Cells seeded in ChronOs ${ }^{\circledR}$ showed a relatively constant amount of newly formed bone inside the scaffolds $\left(\sim 1.5 \mathrm{~mm}^{3}\right)$, as compared to the other two conditions (Fig. 3). Cells seeded in ReproBone ${ }^{\mathrm{TM}}$ showed abundant new bone inside the scaffolds $\left(2.14 \pm 0.58 \mathrm{~mm}^{3}\right)$ when medium dosage of BMP6 was applied, while the low and high dosage of BMP6 led to a marginal amount $\left(\sim 0.68 \mathrm{~mm}^{3}\right)$ of new bone formed inside the scaffolds (Fig. 3). Particularly, for ChronOs ${ }^{\circledR}$ and ReproBone ${ }^{\mathrm{TM}}$ coated with low or medium dosage of BMP6, the bone formed inside the scaffolds was the major contributor to the total bone formation (Fig. 3). In

\section{a}
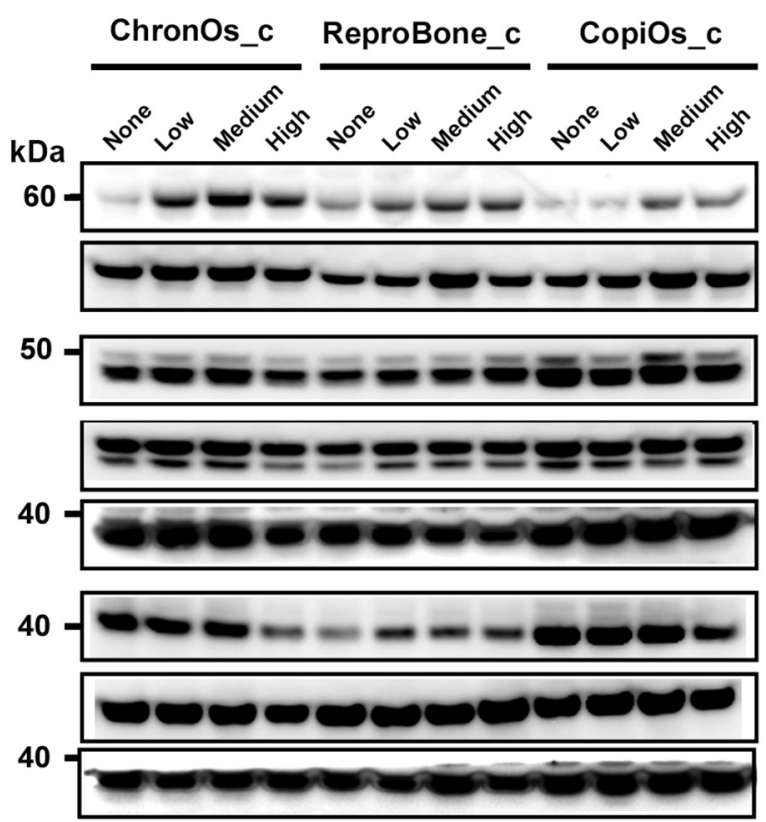

\section{p-SMAD1/5}

SMAD1

p-Erk1/2

Erk1/2

GAPDH

p-p38

p38

GAPDH
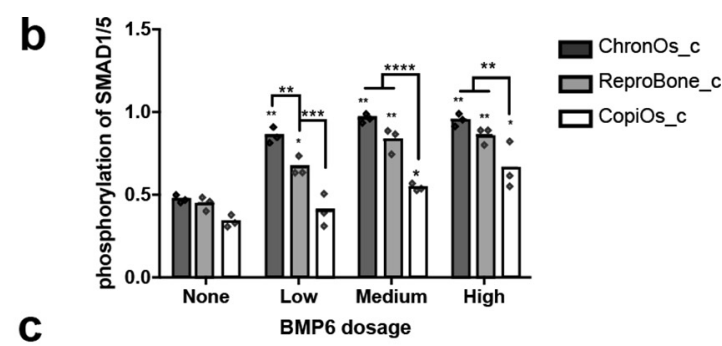

C
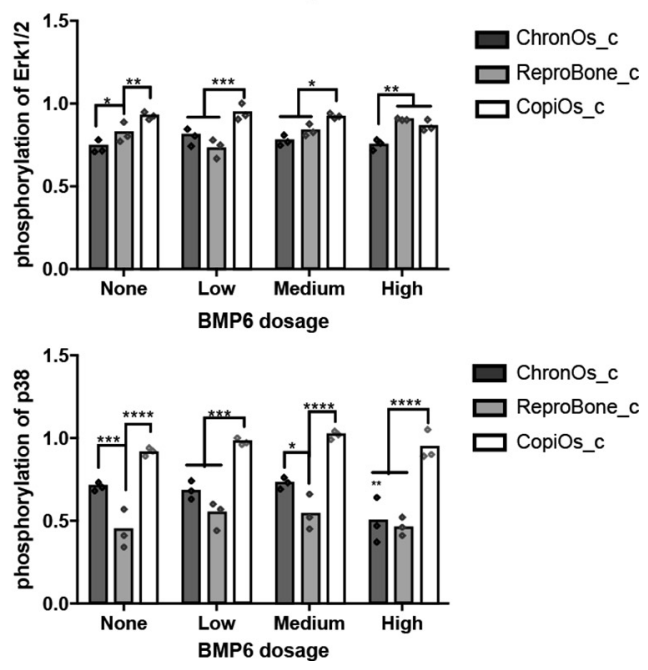

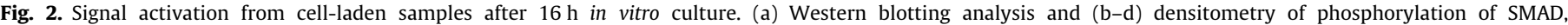

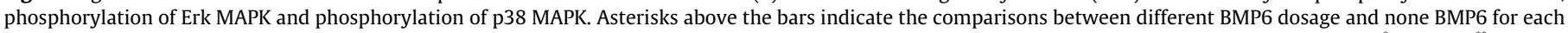

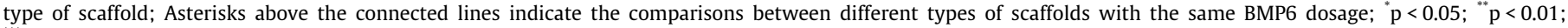
$\mathrm{p}<0.001$. 
a

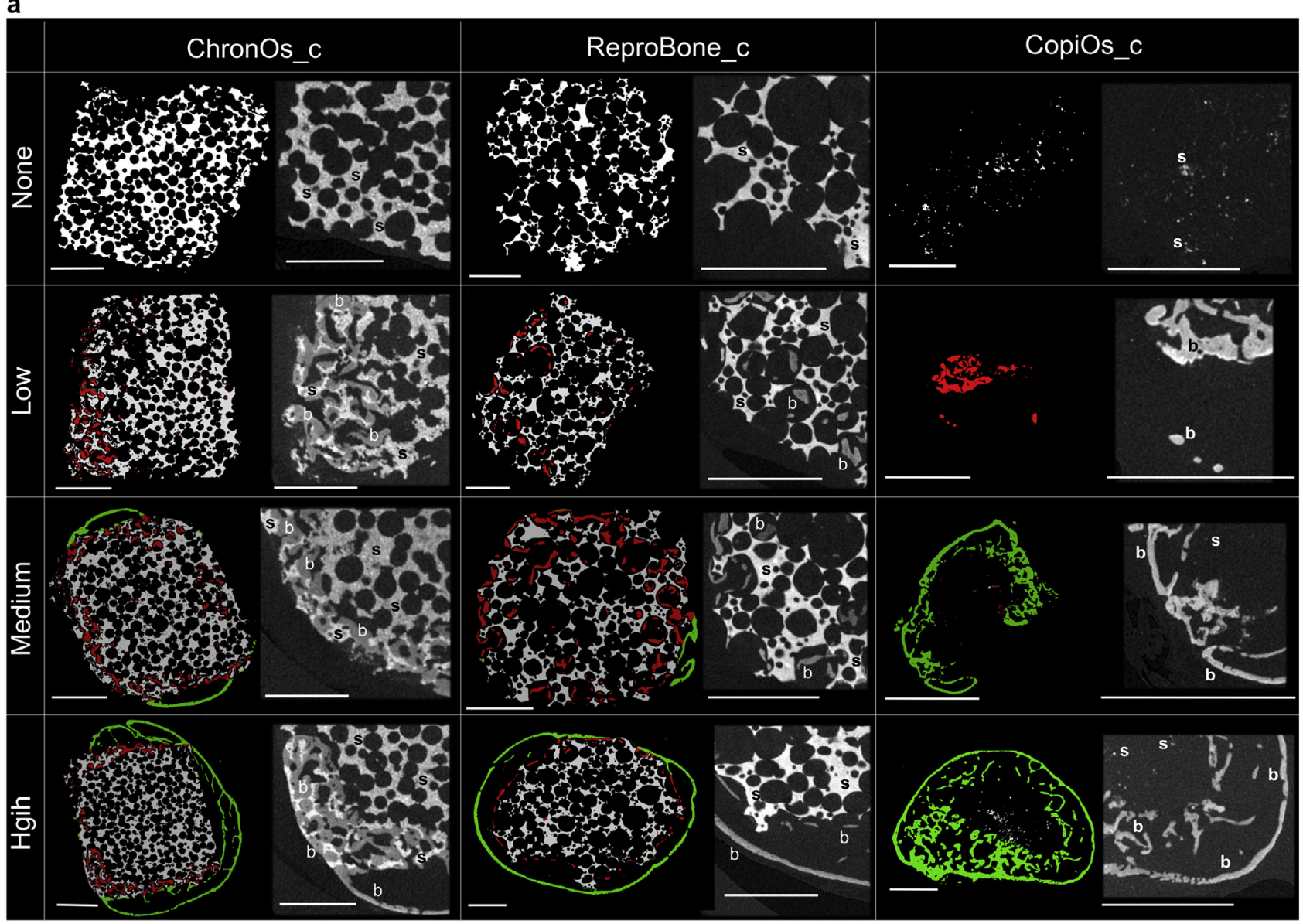

b

5 weeks bone formation

\begin{abstract}
C
\end{abstract}
Bone outside the scaffold d
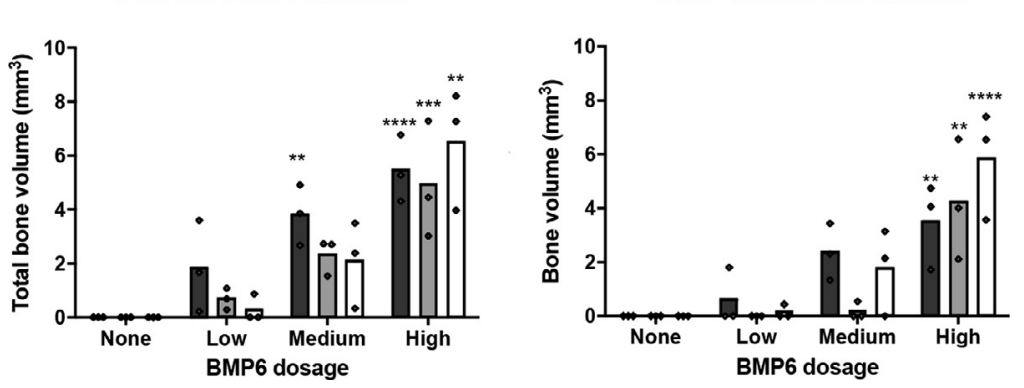

Bone inside the scaffold

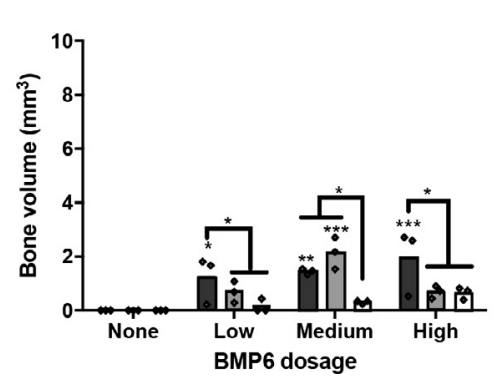

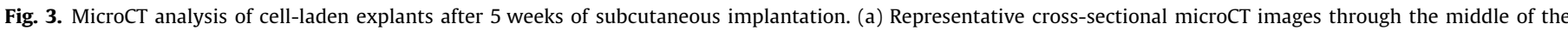

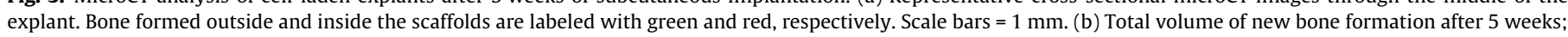

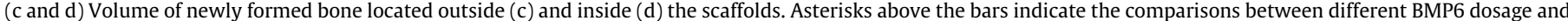

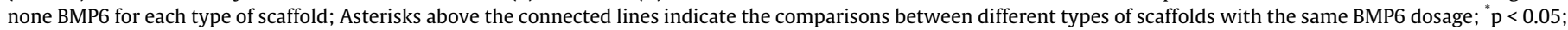
${ }^{* *} \mathrm{p}<0.01 ;{ }^{* * *} \mathrm{p}<0.001$.

contrast, in the case of CopiOs ${ }^{\circledR}$, the majority of new bone was formed outside the scaffold at both medium or high dosage of BMP6 (Fig. 3).

\subsection{General histology}

General histology of 5-week explants was assessed by HE staining. As shown in Fig. 4, hPDCs seeded in bare scaffolds without the addition of BMP6 only showed fibrous tissue infiltration within the explants, although osteoblast-like cells were observed in bare ChronOs $^{\circledR}$ and ReproBone ${ }^{\mathrm{TM}}$. Bone formation was observed when hPDCs seeded in both ChronOs ${ }^{\circledR}$ and ReproBone ${ }^{\mathrm{TM}}$ were combined with BMP6, which confirmed the microCT analysis results. Particularly, the newly formed bone osteoid (indicated as B) was in close contact with the porous surface of scaffolds (indicated as S), which was consistent with those slightly basophilic stained osteoblastlike cells with spherical and large nucleus observed on ChronOs ${ }^{\circledR}$ and ReproBone ${ }^{\mathrm{TM}}$ surfaces at 2 weeks (Fig. S3). Furthermore, when a high dosage of BMP6 was applied, blood vessel formation after 2 weeks (Fig. S3) and an abundant bone marrow formation after 5 weeks (Fig. 4) were observed in the three types of scaffolds seeded with hPDCs.

\subsection{Immunohistochemical staining human specific osteocalcin}

The contribution of the implanted cells to the newly formed bone was assessed by immunohistochemical staining of human specific osteocalcin (OCN) both after 2 weeks and 5 weeks of 


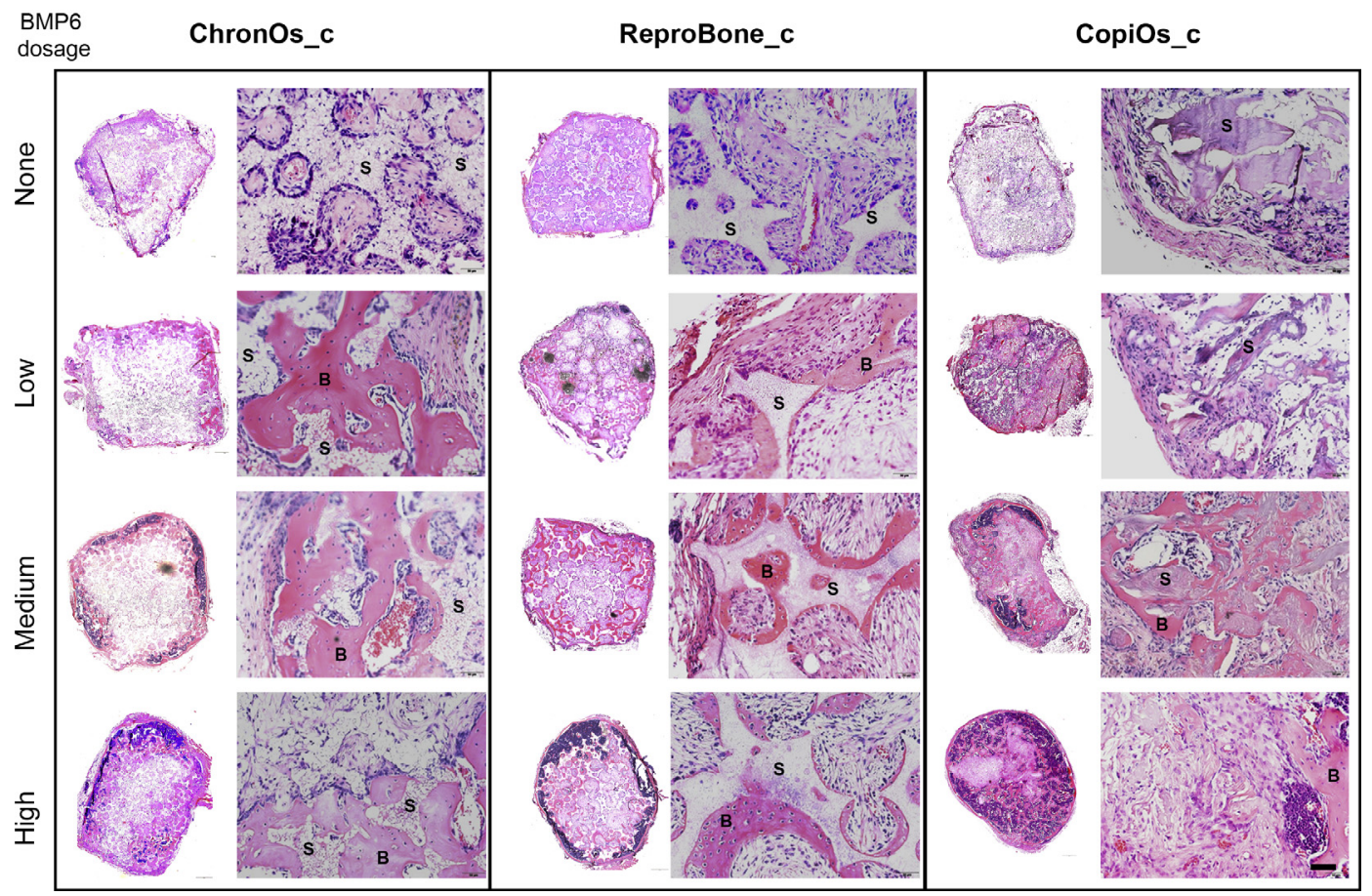

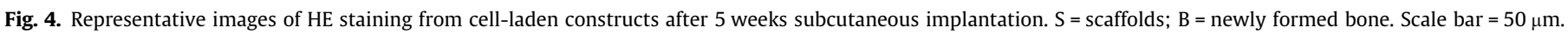

implantation. As shown in Fig. 5, positive human OCN staining, depicted by the brown stain in cell nuclei and newly formed bone matrix was mainly observed within the scaffolds, particularly on the surface of ChronOs ${ }^{\circledR}$ and ReproBone ${ }^{\mathrm{TM}}$ combined with BMP6. The intensity of human OCN stain and staining area increased from 2 weeks to 5 weeks, suggesting the maturation of newly formed bone ossicles. Notably, hPDCs seeded in CopiOs ${ }^{\circledR}$ only showed a very faint positive staining of human OCN after 5 weeks within the scaffolds when high dosage of BMP6 was applied.

\subsection{Multivariate statistical analysis of the empirical data}

In order to decipher the combined effect of $\mathrm{Ca}^{2+}$ release rate and BMP6 dosage, we performed hierarchical clustering of the in vitro cellular response, $\mathrm{Ca}^{2+}$ release rate, and in vivo bone formation. This analysis displayed clustering of phosphorylation of SMAD with the bone volume inside the scaffolds (Fig. 6). Moreover, BMP6 dosage was closely clustered with total bone volume and bone volume outside the scaffolds (Fig. 6). Conversely, phosphorylation of p38 and ERK were closely clustered with $\mathrm{Ca}^{2+}$ release rate, which is directly related to different CaP properties (Fig. 6). Cluster analysis of different combinations of BMP6 dosage and scaffolds properties displayed clustering of cells seeding on ChronOs ${ }^{\circledR}$ and ReproBone ${ }^{\mathrm{TM}}$. A similar combined effect of $\mathrm{BMP}$ and $\mathrm{Ca}^{2+}$ was observed for these two types of cell-seeded scaffolds loaded with the different BMP6 dosages (Fig. 6). In contrast, the hPDCs seeding on CopiOs ${ }^{\circledR}$ with different BMP6 dosage were grouped together, and clearly separated from the other conditions (Fig. 6).

Principle component analysis (PCA) was performed to assess individual and combined effects of the different experimental conditions, as well as to retrieve a maximum amount of information from the entire dataset. The first principal component, which accounts for as much variation in the dataset as possible, is the linear combination of variables that has maximum variance among all linear combinations. The second principal component is the linear combination of variables that accounts for as much of the remaining variation as possible, with the constraint that the correlation between the first and second component is 0 . Upon analysis, different conditions and various biological readouts were separated by factors related to different BMP6 dosage and $\mathrm{Ca}^{2+}$ release rate. Particularly, $\mathrm{Ca}^{2+}$ release was strongly related with p38\&Erk phosphorylation, whereas SMAD phosphorylation was highly correlated with bone volume inside the scaffolds (Fig. 6). Furthermore, $\mathrm{Ca}^{2+}$ release rate and SMAD phosphorylation were observed to have an inverse projection in component 1 , indicating their intrinsic competing effect. On the contrary, in component 2 , BMP6 dosage overrides the effect of $\mathrm{Ca}^{2+}$ release rate and appeared to be a dominant factor for total bone volume (Fig. 6).

\section{Discussion}

The aim of the current study was to comprehensively investigate the combined effect of BMP6 dosage and various types of clinical-grade calcium phosphate scaffolds (with different $\mathrm{Ca}^{2+}$ release rates) on the in vivo ectopic bone forming capacity of hPDC-seeded tissue engineered constructs. We hypothesized that the interaction between BMP6 and physicochemical properties of $\mathrm{CaP}$ scaffolds would affect in vitro cell behavior in terms of metabolic activity and signal pathway activation, hence influencing the in vivo skeletal tissue formation. For this purpose, cell-based constructs were prepared by seeding hPDCs into three different clinical-grades CaP-based scaffolds coated with three dosages of BMP6. The obtained constructs were analyzed for cell metabolic activity and signal activation assays, and then subsequently subcutaneously implanted in immune-deficient mice for 2 weeks and 5 weeks. Our main finding is that the interactions between CaP properties and BMP6 affect in vitro cell behavior and influencing the in vivo biological performance.

In this study, hPDCs showed different glycolysis pathways after 16 hrs of seeding in the three types of clinical-grade CaP scaffolds, which have a clear difference in morphological structure as well and $\mathrm{Ca}^{2+}$ release rate. Specifically, ChronOs ${ }^{\circledR}$ and ReproBone ${ }^{\mathrm{TM}}$ have a medium and slow $\mathrm{Ca}^{2+}$ release rate, and $\mathrm{CopiOs}^{\circledR}$ has a fast $\mathrm{Ca}^{2+}$ release rate, as both measured in vitro [27] and in vivo [21]. Cells 

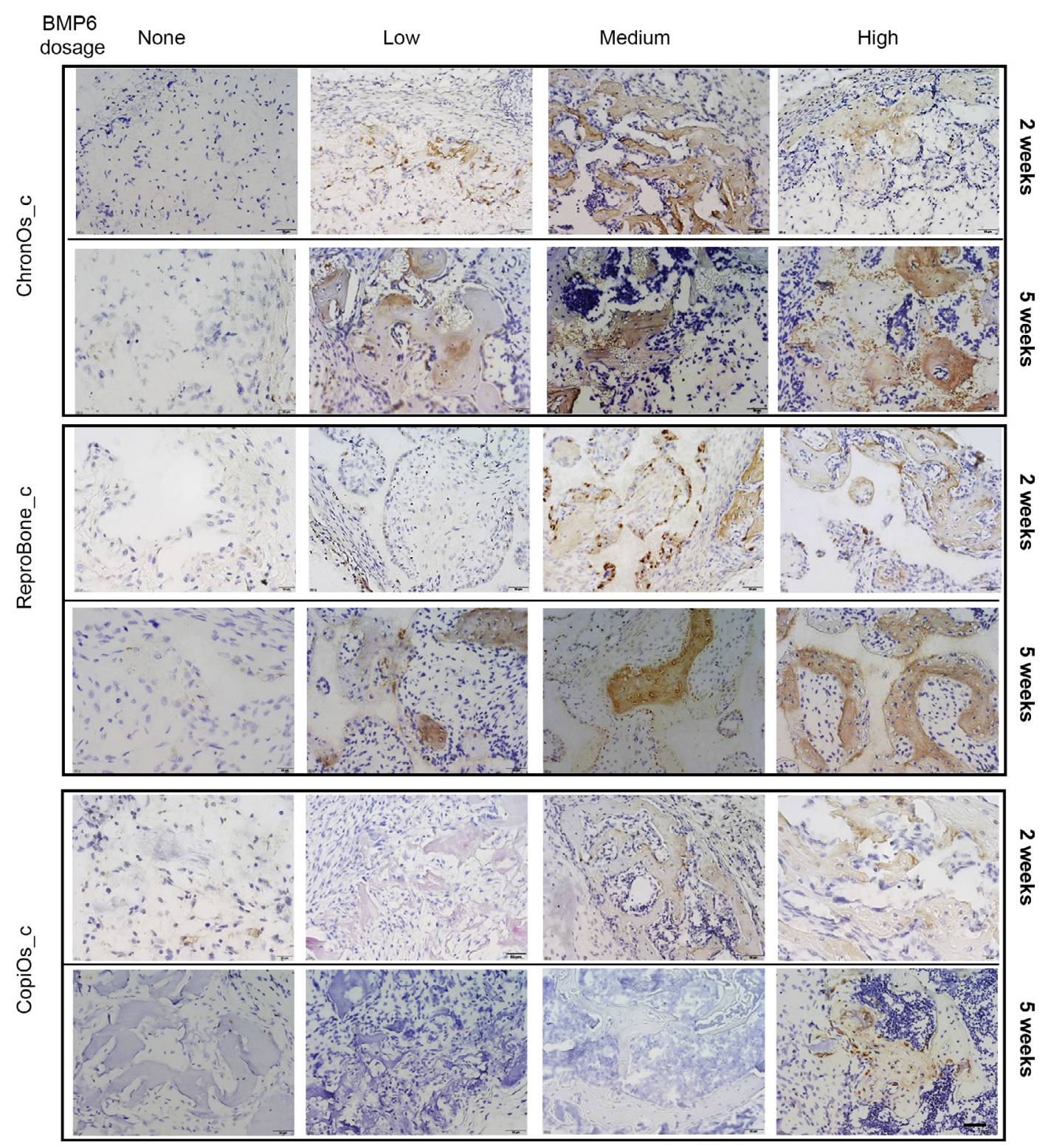

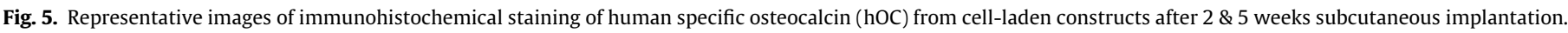
Scale bar $=50 \mu \mathrm{m}$.

seeded in ChronOs ${ }^{\circledR}$ displayed an anaerobic glycolysis, where one glucose molecule generated almost two lactate molecules. In contrast, cells seeded in the other two types of scaffolds with higher porosity showed less lactate generation compared to the ones in ChronOs $^{\circledR}$, indicating that an aerobic glycolysis (Warburg Effect) was predominant [28]. Anaerobic glycolysis is preferred by the differentiated cells when oxygen is limited, whereas the proliferating cells shift their metabolic machinery toward to aerobic glycolysis (Warburg Effect) with high levels of glucose uptake and lactate production [29]. In our previous study, ChronOs ${ }^{\circledR}$ was shown to have the lowest porosity (about 60\%) among the three types of scaffolds [21]. The low porosity along with its medium $\mathrm{Ca}^{2+}$ release rate might result in a limited oxygen access within the ChronOs ${ }^{\circledR}$ constructs. In contrast, the high porosity (about 90\%) along with the presence of Type I collagen matrix in CopiOs ${ }^{\circledR}$ might be favoring cell proliferation [30].

It is known that hPDCs express BMP Type I receptors (ALK3 \& ALK6) and Type II receptors (BMPR2) [26], which are binding the BMP6 ligand [31]. Upon seeding into CaP scaffolds, hPDCs showed an evident phosphorylation of p38 and ERK from all conditions regardless of BMP6 dosage and scaffold type. ERK and p38activated MAPK pathways can be initiated by both non-canonical BMP pathways and $\mathrm{Ca}^{2+}$ signals $[32,33]$. Yet, our data showed that the phosphorylation of p38 and ERK was independent of BMP6 dosage, suggesting that both $\mathrm{p} 38$ and ERK-MAPK pathways were dominated by $\mathrm{Ca}^{2+}$ signals. Moreover, the chemical composition of CaP strongly affected the signal amplitude of p38 and ERKMAPK. Cells seeded in CopiOs ${ }^{\circledR}$ (diphasic calcium phosphate) showed the highest phosphorylation of p38 and ERK above ChronOs $^{\circledR}(\beta-\mathrm{TCP})$ and ReproBone ${ }^{\mathrm{TM}}$ (mixture of $\beta$-TCP and hydroxyapatite). Diphasic calcium phosphate was shown to have a significantly faster $\mathrm{Ca}^{2+}$ release than $\beta$-TCP and hydroxyapatite [21], and we assume the faster $\mathrm{Ca}^{2+}$ release might result in a higher cumulative $\mathrm{Ca}^{2+}$ signaling to the hPDCs seeded in CopiOs ${ }^{\circledR}$, hence leading to a significantly greater activation of downstream p38 and ERKMAPK pathways.

When BMP6 was present, hPDCs seeded in CaP scaffolds showed an evident phosphorylation of SMAD1/5/8, indicating the 
a

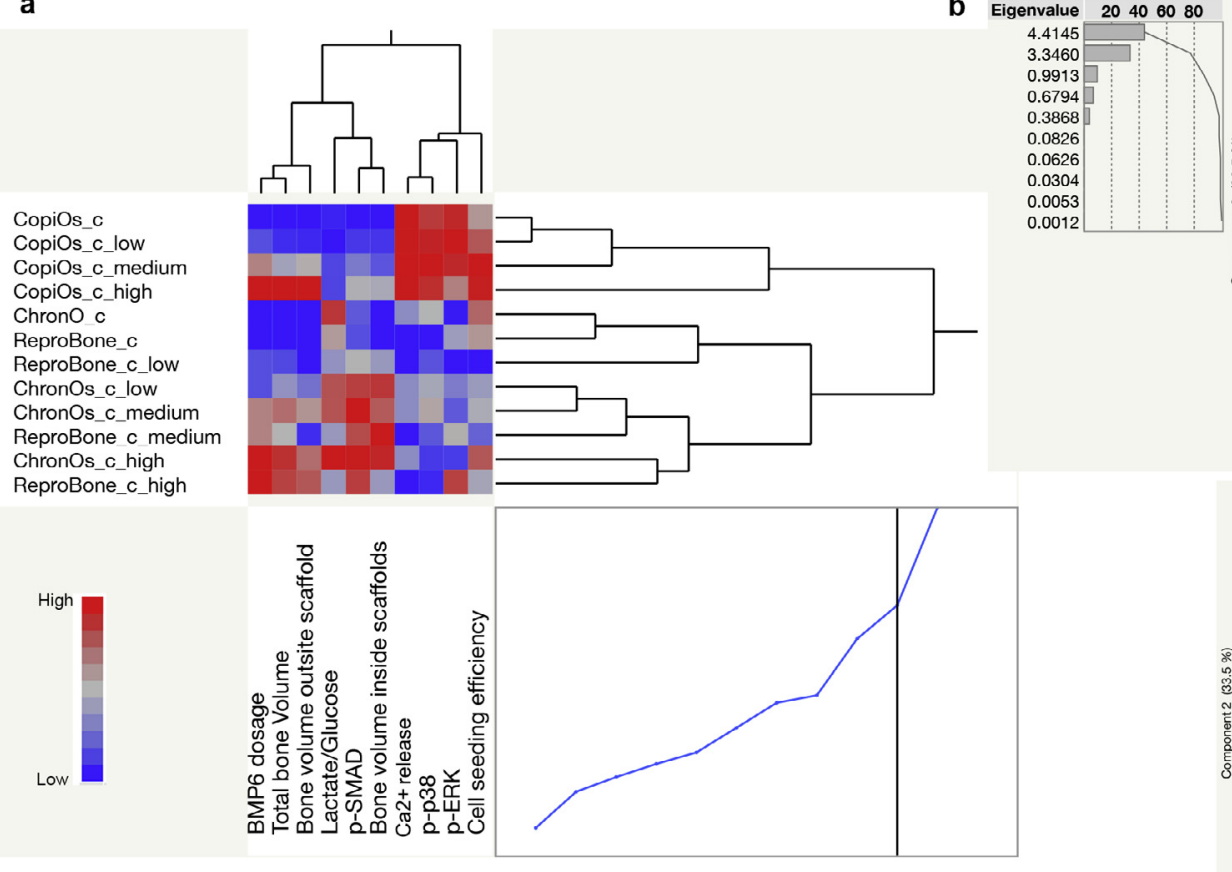

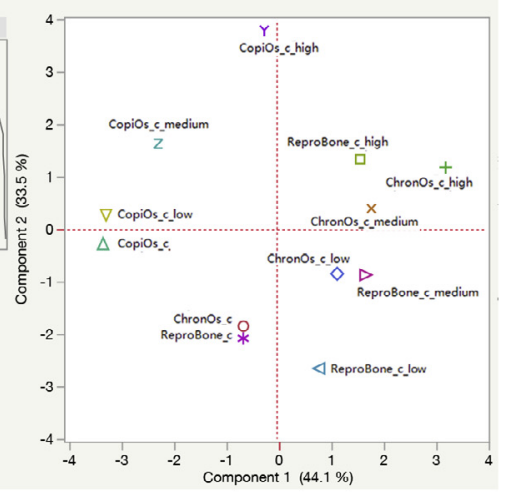

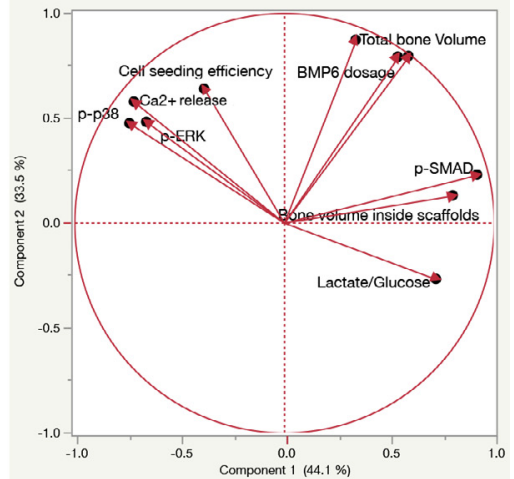

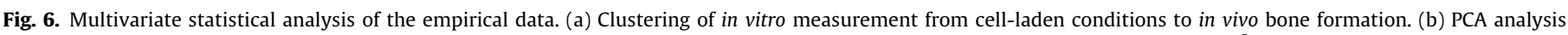

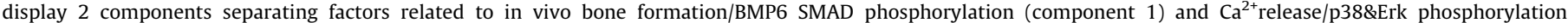
(Component 2).

canonical SMAD-dependent pathway activation [34]. Also, the SMAD1/5/8 signal amplitude gradually increased with the increments of BMP6 doses, which correlates with earlier experiments and modeling analyses of dynamics of TGF- $\beta$ signaling [35]. When the same dosage of BMP6 was applied, the SMAD1/5/8 signal amplitude showed a tendency to decrease along with the increase of $\mathrm{Ca}^{2+}$ driven p38 and ERK phosphorylation, suggesting a crosstalk between canonical BMP pathway and $\mathrm{Ca}^{2+}$ signaling. BMP signaling pathways are strictly regulated by cross-talk and feedback-loops, leading to a controlled down-stream osteogenic process upon accurate pathway activation [36]. In consequence, the inappropriate $\mathrm{Ca}^{2+}$ signal activation may inhibit the activated canonical BMP signaling, leading to the decreased sensitivity to the low dosage of BMP6.

In this study, bone formation after 5 weeks of subcutaneous implantation was indeed observed with hPDCs seeded on BMP6 coated CaP scaffolds. Remarkably, in all three types of scaffolds, the total volume of newly formed bone increased along with the increasing BMP6 dosage. Such phenomenon suggests that BMP6 amplifies the stimulatory effect of CaP-scaffolds and acts as an important factor in driving bone formation, which corroborates with our previous study [26]. Furthermore, the increased BMP6 dosage resulted in an amplified volume of new bone outside the scaffolds, which is mainly formed by host cells. This might be caused by BMP6 diffusion from the scaffolds, which attracts host cells to proliferate and ossify at the peripheral site. In contrast, new bone formed inside the scaffold, which is primarily contributed by implanted cells evidenced by positive staining of human specific osteocalcin, is mainly dependent on material properties instead of BMP6 dosage. This suggests that the combined effect of BMP6 and CaP-stimulation of hPDCs resulted in new bone formation inside the scaffold following the intramembranous pathway [37]. The bone formation within the scaffolds at early time points is important for clinical translation as such interconnected spongy bone structure is crucial to provides a stable structure for the fracture during the soft callus stage [38].

Considering the large and complex set of our empirical data, we applied multivariate statistical methods in order to gain further insight into the combined effects of multiple factors in the experimental design. Possibly, the BMP6-CaP scaffold interaction and the subsequent varied in vivo tissue formation could be caused by different downstream signaling activation. Interestingly, BMP6 dosage was clustered to total bone volume and bone volume outside scaffolds, while bone volume inside scaffolds and the elevated phosphorylation of SMAD1/5/8 were grouped together. This suggests the importance of appropriate activation of canonical BMP signaling pathway in hPDCs/BMP6/CaP constructs as the crucial driving force for cell differentiation and bone formation [39].

Due to their distinctively different chemical composition, the three types of CaP scaffolds in the current study were shown to have different $\mathrm{Ca}^{2+}$ release rate, namely $\mathrm{CopiOs}^{\circledR}>\mathrm{ChronOs}^{\circledR}>$ ReproBone $^{\mathrm{TM}}$ [21]. As aforementioned, the cross-talk between canonical BMP pathway and $\mathrm{Ca}^{2+}$ signaling potentially influences the in vivo bone formation $[18,26]$. Therefore, the cluster analysis also allows us to understand the weight value of the CaP scaffold characteristics with regard to the in vitro cellular behavior and the in vivo outcome when combined with different dosages of BMP6. We show herein that when low or medium dosage of BMP6 was applied, cell-laden CopiOs ${ }^{\circledR}$ constructs were clustered to the cell-free CopiOs $^{\circledR}$ scaffolds, distinctively separated from the ChronOs ${ }^{\circledR}$ and Reprobone ${ }^{\mathrm{TM}}$. This proposes that the fast $\mathrm{Ca}^{2+}$ release to be dominant over the low or medium dose of BMP6 stimulation on hPDCs, which is further supported by the PCA analysis. Concomitantly, cells seeding on these three types of scaffolds 
combined with high dosage of BMP6 were clustered together, indicating the saturation of BMP6 is overriding the CaP scaffold material properties.

To conclude, in this study, we have developed hPDCs-based bone tissue engineering constructs by combining the stimulatory effects of BMP6 and CaP scaffolds. We have been able to decipher the individual and combined effect of BMP6 dosage and $\mathrm{Ca}^{2+}$ release rate on the in vitro and in vivo behavior of hPDCs/BMP6/ $\mathrm{CaP}$ combination constructs. Cells seeded on CaP scaffolds with intermediate $\mathrm{Ca}^{2+}$ release rate combined with low or medium dosage of BMP6 demonstrated a robust new ectopic bone formation after 5 weeks contributed by both donor and host cells. This phenomenon might be due to the delicate balance between $\mathrm{Ca}^{2+}$ and BMP pathways, allowing an appropriate activation of the canonical BMP signaling pathway that is required for in vivo bone formation. Our findings illustrate the importance of matching BMP6 dosage and CaP properties in development of hPDCs/ BMP6/CaP combination constructs. It also provides insights with regards to clinical translation of such cell-based constructs for bone regeneration.

\section{Acknowledgments}

This work was supported by the European Research Council grants under the European Union's Seventh Framework Program (FP/2007-2013) (ERC Grant Agreements No. 294191 \& 279100) and Hercules Foundation (Project AKUL09/001). Dr. Wei Ji and Dr. Greet Kerckhofs were financed by the postdoctoral grant of the Fonds Wetenschappelijk Onderzoek (FWO 12G2718N and FWO 12R4315N). The authors would like to acknowledge Dr. Yoke Chin Chai for insightful discussions and critical suggestions. The authors would also like to thank Ms. Kathleen Bosmans Mr. Stijn Bellinkx, and Mr. Sven Van Camp (Katholieke Hogeschool Limburg, Belgium)for their assistance during animal surgeries, and histological preparation of the samples, respectively. This work is part of Prometheus, the KU Leuven Research \& Development division of Skeletal Tissue Engineering (www.kuleuven.be/prometheus).

\section{Conflict of interests}

The authors declare that there is no conflict of interests.

\section{Author contributions}

W. Ji designed \& planned the experiment, performed data collection $\&$ analysis, as well as manuscript preparation and editing. G. Kerckhofs designed \& planned the experiment, performed data collection \& analysis, as well as manuscript editing. C. Geeroms performed data analysis and manuscript editing. M. Marechal planned the experiment and performed manuscript editing. F.P. Luyten and L. Geris supervised the experiments, and assisted with the data interpretation and manuscript editing.

\section{Appendix A. Supplementary data}

Supplementary data to this article can be found online at https://doi.org/10.1016/j.actbio.2018.09.046.

\section{References}

[1] T. Meling, K. Harboe, K. Soreide, Incidence of traumatic long-bone fractures requiring in-hospital management: a prospective age- and gender-specific analysis of 4890 fractures, Injury 40 (11) (2009) 1212-1219.

[2] J.A. Buza, T. Einhorn, Bone healing in 2016, Clin. Cases Miner. Bone 13 (2) (2016) 101-105.
[3] W.L. Grayson, B.A. Bunnell, E. Martin, T. Frazier, B.P. Hung, J.M. Gimble, Stromal cells and stem cells in clinical bone regeneration, Nat. Rev. Endocrinol. 11 (3) (2015) 140-150.

[4] L.A. Mills, A.H. Simpson, The relative incidence of fracture non-union in the Scottish population (5.17 million): a 5-year epidemiological study, BMJ Open 3 (2) (2013)

[5] D. Holmes, Non-union bone fracture: a quicker fix, Nature 550 (7677) (2017) S193.

[6] K. Niedzielski, M. Synder, The treatment of pseudarthrosis using the Ilizarov method, Ortop Traumatol. Rehabil. 2 (3) (2000) 46-48.

[7] C. Loebel, J.A. Burdick, Engineering stem and stromal cell therapies for musculoskeletal tissue repair, Cell Stem Cell 22 (3) (2018) 325-339.

[8] W. Ji, J. Bolander, Y.C. Chai, H. Katagiri, M. Marechal, F.P. Luyten, Toward advanced therapy medicinal products (ATMPs) combining bone morphogenetic proteins (BMP) and cells for bone regeneration, in: S. Vukicevic, K.T. Sampath (Eds.), Bone Morphogenetic Proteins: Systems Biology Regulators, Springer, Cham, 2017, pp. 127-169.

[9] M. Ollivier, A.M. Gay, A. Cerlier, A. Lunebourg, J.N. Argenson, S. Parratte, Can we achieve bone healing using the diamond concept without bone grafting for recalcitrant tibial nonunions?, Injury 46 (7) (2015) 1383-1388

[10] C. Colnot, Skeletal cell fate decisions within periosteum and bone marrow during bone regeneration, J. Bone Miner. Res. 24 (2) (2009) 274-282.

[11] S.J. Roberts, N. van Gastel, G. Carmeliet, F.P. Luyten, Uncovering the periosteum for skeletal regeneration: the stem cell that lies beneath, Bone 70 (2015) 1018.

[12] C. De Bari, F. Dell'Accio, F. P Luyten, Human periosteum-derived cells maintain phenotypic stability and chondrogenic potential throughout expansion regardless of donor age, Arthritis Rheum. 44 (1) (2001) 85-95.

[13] J. Leijten, Y.C. Chai, I. Papantoniou, L. Geris, J. Schrooten, F.P. Luyten, Cell based advanced therapeutic medicinal products for bone repair: Keep it simple?, Adv Drug Delivery Rev. 84 (2015) 30-44.

[14] O. Duchamp de Lageneste, A. Julien, R. Abou-Khalil, G. Frangi, C. Carvalho, N Cagnard, C. Cordier, S.J. Conway, C. Colnot, Periosteum contains skeletal stem cells with high bone regenerative potential controlled by Periostin, Nat. Commun. 9 (1) (2018) 773.

[15] Y.C. Chai, A. Carlier, J. Bolander, S.J. Roberts, L. Geris, J. Schrooten, H. Van Oosterwyck, F.P. Luyten, Current views on calcium phosphate osteogenicity and the translation into effective bone regeneration strategies, Acta Biomater. 8 (11) (2012) 3876-3887.

[16] H.H. Xu, P. Wang, L. Wang, C. Bao, Q. Chen, M.D. Weir, L.C. Chow, L. Zhao, X Zhou, M.A. Reynolds, Calcium phosphate cements for bone engineering and their biological properties, Bone Res. 5 (2017) 17056

[17] I. Denry, L.T. Kuhn, Design and characterization of calcium phosphate ceramic scaffolds for bone tissue engineering, Dent. Mater. 32 (1) (2016) 43-53.

[18] J. Bolander, Y.C. Chai, L. Geris, J. Schrooten, D. Lambrechts, S.J. Roberts, F.P. Luyten, Early BMP, Wnt and $\mathrm{Ca}(2+) / \mathrm{PKC}$ pathway activation predicts the bone forming capacity of periosteal cells in combination with calcium phosphates, Biomaterials 86 (2016) 106-118.

[19] Y.C. Chai, S.J. Roberts, E. Desmet, G. Kerckhofs, N. van Gastel, L. Geris, G. Carmeliet, J. Schrooten, F.P. Luyten, Mechanisms of ectopic bone formation by human osteoprogenitor cells on CaP biomaterial carriers, Biomaterials 33 (11) (2012) 3127-3142.

[20] S.J. Roberts, L. Geris, G. Kerckhofs, E. Desmet, J. Schrooten, F.P. Luyten, The combined bone forming capacity of human periosteal derived cells and calcium phosphates, Biomaterials 32 (19) (2011) 4393-4405.

[21] G. Kerckhofs, Y.C. Chai, F.P. Luyten, L. Geris, Combining microCT-based characterization with empirical modelling as a robust screening approach for the design of optimized CaP-containing scaffolds for progenitor cellmediated bone formation, Acta Biomater. 35 (2016) 330-340.

[22] G.B. Bishop, T.A. Einhorn, Current and future clinical applications of bone morphogenetic proteins in orthopaedic trauma surgery, Int. Orthop. 31 (6) (2007) 721-727.

[23] A.P. White, A.R. Vaccaro, J.A. Hall, P.G. Whang, B.C. Friel, M.D. McKee, Clinical applications of BMP-7/OP-1 in fractures, nonunions and spinal fusion, Int. Orthop. 31 (6) (2007) 735-741.

[24] S. Vukicevic, H. Oppermann, D. Verbanac, M. Jankolija, I. Popek, J. Curak, J. Brkljacic, M. Pauk, I. Erjavec, I. Francetic, I. Dumic-Cule, M. Jelic, D. Durdevic, T Vlahovic, R. Novak, V. Kufner, T. Bordukalo Niksic, M. Kozlovic, Z. Banic Tomisic, J. Bubic-Spoljar, I. Bastalic, S. Vikic-Topic, M. Peric, M. Pecina, L. Grgurevic, The clinical use of bone morphogenetic proteins revisited: a novel biocompatible carrier device OSTEOGROW for bone healing, Int. Orthop. 38 (3) (2014) 635-647.

[25] S.N. Lissenberg-Thunnissen, D.J. de Gorter, C.F. Sier, I.B. Schipper, Use and efficacy of bone morphogenetic proteins in fracture healing, Int. Orthop. 35 (9) (2011) 1271-1280.

[26] J. Bolander, W. Ji, L. Geris, V. Bloemen, Y.C. Chai, J. Schrooten, F.P. Luyten, The combined mechanism of bone morphogenetic protein- and calcium phosphate-induced skeletal tissue formation by human periosteum derived cells, Eur. Cell Mater. 31 (2016) 11-25.

[27] V. Manhas, Y. Guyot, G. Kerckhofs, Y.C. Chai, L. Geris, Computational modelling of local calcium ions release from calcium phosphate-based scaffolds, Biomech. Model. Mech. 16 (2) (2017) 425-438.

[28] O. Warburg, On the origin of cancer cells, Science 123 (3191) (1956) 309-314

[29] M.G. Vander Heiden, L.C. Cantley, C.B. Thompson, Understanding the Warburg effect: the metabolic requirements of cell proliferation, Science 324 (5930) (2009) 1029-1033. 
[30] K.S. Tsai, S.Y. Kao, C.Y. Wang, Y.J. Wang, J.P. Wang, S.C. Hung, Type I collagen promotes proliferation and osteogenesis of human mesenchymal stem cells via activation of ERK and Akt pathways, J. Biomed. Mater. Res. A 94 (3) (2010) 673-682.

[31] P.C. Bessa, M. Casal, R.L. Reis, Bone morphogenetic proteins in tissue engineering: the road from the laboratory to the clinic, part I (basic concepts), J. Tissue Eng. Regene. Med. 2 (1) (2008) 1-13.

[32] M.S. Rahman, N. Akhtar, H.M. Jamil, R.S. Banik, S.M. Asaduzzaman, TGF-beta/ BMP signaling and other molecular events: regulation of osteoblastogenesis and bone formation, Bone Res. 3 (2015) 15005.

[33] N. Agell, O. Bachs, N. Rocamora, P. Villalonga, Modulation of the Ras/Raf/MEK ERK pathway by $\mathrm{Ca}(2+)$, and calmodulin, Cell Signallin 14 (8) (2002) 649-654.

[34] R.N. Wang, J. Green, Z. Wang, Y. Deng, M. Qiao, M. Peabody, Q. Zhang, J. Ye, Z. Yan, S. Denduluri, O. Idowu, M. Li, C. Shen, A. Hu, R.C. Haydon, R. Kang, J. Mok,
M.J. Lee, H.L. Luu, L.L. Shi, Bone Morphogenetic Protein (BMP) signaling in development and human diseases, Genes Dis. 1 (1) (2014) 87-105.

[35] Z. Zi, D.A. Chapnick, X. Liu, Dynamics of TGF-beta/Smad signaling, FEBS Lett. 586 (14) (2012) 1921-1928.

[36] J. Groppe, J. Greenwald, E. Wiater, J. Rodriguez-Leon, A.N. Economides, W. Kwiatkowski, M. Affolter, W.W. Vale, J.C. Izpisua Belmonte, S. Choe, Structural basis of BMP signalling inhibition by the cystine knot protein Noggin, Nature 420 (6916) (2002) 636-642.

[37] R. Marsell, T.A. Einhorn, The biology of fracture healing, Injury 42 (6) (2011) $551-555$.

[38] D. Holmes, Closing the gap, Nature 550 (7677) (2017) S194-S195.

[39] S. Vukicevic, L. Grgurevic, BMP-6 and mesenchymal stem cell differentiation, Cytokine Growth Factor Rev. 20 (5-6) (2009) 441-448. 